\title{
System Size, Energy, Pseudorapidity, and Centrality Dependence of Elliptic Flow
}

B. Alver, ${ }^{4}$ B. B. Back, ${ }^{1}$ M. D. Baker, ${ }^{2}$ M. Ballintijn, ${ }^{4}$ D. S. Barton, ${ }^{2}$ R. R. Betts, ${ }^{6}$ A. A. Bickley, ${ }^{7}$ R. Bindel, ${ }^{7}$ W. Busza,${ }^{4}$ A. Carroll, ${ }^{2}$ Z. Chai, ${ }^{2}$ V. Chetluru, ${ }^{6}$ M. P. Decowski, ${ }^{4}$ E. García, ${ }^{6}$ T. Gburek, ${ }^{3}$ N. George, ${ }^{2}$ K. Gulbrandsen, ${ }^{4}$ C. Halliwell, ${ }^{6}$ J. Hamblen, ${ }^{8}$ I. Harnarine, ${ }^{6}$ M. Hauer, ${ }^{2}$ C. Henderson, ${ }^{4}$ D. J. Hofman, ${ }^{6}$ R. S. Hollis, ${ }^{6}$ R. Hołyński, ${ }^{3}$ B. Holzman, ${ }^{2}$ A. Iordanova, ${ }^{6}$ E. Johnson, ${ }^{8}$ J. L. Kane, ${ }^{4}$ N. Khan,${ }^{8}$ P. Kulinich, ${ }^{4}$ C. M. Kuo,${ }^{5}$ W. Li, ${ }^{4}$ W. T. Lin,${ }^{5}$ C. Loizides ${ }^{4}$ S. Manly, ${ }^{8}$ A. C. Mignerey, ${ }^{7}$ R. Nouicer, ${ }^{2}$ A. Olszewski, ${ }^{3}$ R. Pak, ${ }^{2}$ C. Reed, ${ }^{4}$ E. Richardson, ${ }^{7}$ C. Roland, ${ }^{4}$ G. Roland,${ }^{4}$ J. Sagerer, ${ }^{6}$ H. Seals, ${ }^{2}$ I. Sedykh, ${ }^{2}$ C. E. Smith, ${ }^{6}$ M. A. Stankiewicz, ${ }^{2}$ P. Steinberg, ${ }^{2}$ G. S. F. Stephans, ${ }^{4}$ A. Sukhanov, ${ }^{2}$ A. Szostak, ${ }^{2}$ M. B. Tonjes ${ }^{7}$ A. Trzupek,${ }^{3}$ C. Vale,${ }^{4}$ G. J. van Nieuwenhuizen, ${ }^{4}$ S. S. Vaurynovich, ${ }^{4}$ R. Verdier,${ }^{4}$ G. I. Veres, ${ }^{4}$ P. Walters, ${ }^{8}$ E. Wenger, ${ }^{4}$ D. Willhelm, ${ }^{7}$ F. L. H. Wolfs, ${ }^{8}$ B. Wosiek, ${ }^{3}$ K. Woźniak, ${ }^{3}$ S. Wyngaardt, ${ }^{2}$ and B. Wysłouch ${ }^{4}$

${ }^{1}$ Physics Division, Argonne National Laboratory, Argonne, Illinois 60439-4843, USA

${ }^{2}$ Chemistry and C-A Departments, Brookhaven National Laboratory, Upton, New York 11973-5000, USA

${ }^{3}$ Institute of Nuclear Physics PAN, Kraków, Poland

${ }^{4}$ Laboratory for Nuclear Science, Massachusetts Institute of Technology, Cambridge, Massachusetts 02139-4307, USA

${ }^{5}$ Department of Physics, National Central University, Chung-Li, Taiwan

${ }^{6}$ Department of Physics, University of Illinois at Chicago, Chicago, Illinois 60607-7059, USA

${ }^{7}$ Department of Chemistry, University of Maryland, College Park, Maryland 20742, USA

${ }^{8}$ Department of Physics and Astronomy, University of Rochester, Rochester, New York 14627, USA

(Received 21 November 2006; published 15 June 2007)

This Letter presents measurements of the elliptic flow of charged particles as a function of pseudorapidity and centrality from $\mathrm{Cu}-\mathrm{Cu}$ collisions at 62.4 and $200 \mathrm{GeV}$ using the PHOBOS detector at the Relativistic Heavy Ion Collider. The elliptic flow in $\mathrm{Cu}-\mathrm{Cu}$ collisions is found to be significant even for the most central events. For comparison with the Au-Au results, it is found that the detailed way in which the collision geometry (eccentricity) is estimated is of critical importance when scaling out system-size effects. A new form of eccentricity, called the participant eccentricity, is introduced which yields a scaled elliptic flow in the $\mathrm{Cu}-\mathrm{Cu}$ system that has the same relative magnitude and qualitative features as that in the Au-Au system.

DOI: 10.1103/PhysRevLett.98.242302

PACS numbers: $25.75 .-\mathrm{q}$

The characterization of the collective flow of produced particles by their azimuthal anisotropy has proven to be one of the more fruitful probes of the dynamics in $\mathrm{Au}-\mathrm{Au}$ collisions at the Relativistic Heavy Ion Collider (RHIC) [1-8]. Elliptic flow, which is related to the initial spatial shape of the produced matter, has been of particular interest, as it can provide much information about the degree of thermalization of the hot, dense medium. It is, therefore, beneficial to study flow in smaller collision systems, such as $\mathrm{Cu}-\mathrm{Cu}$ which has one-third the number of nucleons in $\mathrm{Au}-\mathrm{Au}$. Exactly how flow scales between collision systems (e.g., a simple scaling with system size or geometry, constituent quarks or transverse momentum, or something more complex [9-11]) is crucial to the understanding of the properties of the produced matter. The dependence of elliptic flow on the geometry of the collision is of particular importance, as flow is thought to depend heavily on the initial spatial anisotropy. Additionally, any fluctuations would be expected to have more of an effect in a smaller system.

Elliptic flow has been studied extensively in $\mathrm{Au}-\mathrm{Au}$ collisions at RHIC as a function of pseudorapidity $(\eta)$, centrality, transverse momentum, and energy [1-8]. The large pseudorapidity coverage of the PHOBOS detector makes it ideally suited for probing the longitudinal structure of the collision, the dynamics of which have only recently begun to be understood away from midrapidity [12]. This work presents new results on data taken by the PHOBOS experiment at RHIC showing a detailed comparison of differential measurements of elliptic flow in $\mathrm{Cu}$ $\mathrm{Cu}$ and $\mathrm{Au}-\mathrm{Au}$ collisions at $\sqrt{s_{N N}}=62.4$ and $200 \mathrm{GeV}$.

The strength of the elliptic flow $\boldsymbol{v}_{2}$ is usually defined as the coefficient of the second harmonic in the Fourier expansion of the particle azimuthal angle distribution relative to the $A-A$ reaction plane $\Psi_{R}$. For this analysis $\Psi_{2}$, an estimate of $\Psi_{R}$ based on the produced particles, was used as in Ref. [13]. The implications of this will be examined in the text.

The PHOBOS detector is composed of silicon pad detectors for tracking, vertex detection, and multiplicity measurements. Details of the setup and the layout of the silicon sensors can be found elsewhere [14]. Key elements of the detector used in this analysis include the first six layers of both silicon spectrometer arms, the silicon vertex detector (VTX), the silicon octagonal multiplicity detector (OCT), three annular silicon multiplicity detectors to either side of the collision point, and two sets of scintillating paddle counters for centrality determination. 
Monte Carlo simulations of the detector performance were based on the HIJING event generator [15] and the GEANT 3.211 [16] simulation package, folding in the signal response for scintillator counters and silicon sensors.

The data shown here were taken with the PHOBOS detector at RHIC during the years 2001-2005. The Au$\mathrm{Au}$ data are published in previous papers describing work at $\sqrt{s_{N N}}=19.6,62.4,130$, and $200 \mathrm{GeV}$ [1-3]. The $\mathrm{Cu}-\mathrm{Cu}$ data at $\sqrt{s_{N N}}=62.4$ and $200 \mathrm{GeV}$ presented here were analyzed in a similar fashion, using the hit-based method that utilized hits in the VTX, OCT, and ring subdetectors to measure flow over a wide range in pseudorapidity $(|\eta|<$ 5.4) and a track-based method that made use of tracks in the spectrometer arms and had a smaller pseudorapidity coverage $(0.0<\eta<1.0)$. For details on the hit-based and track-based methods, see Refs. [1,2], respectively.

The event-by-event collision vertex was determined using the intersection of tracks identified in the VTX and extrapolated back to a common point. The flow analysis was based on the anisotropy of the azimuthal distribution of charged particles traversing the detector. At the points where charged tracks passed through an active silicon detector, energy was deposited in the form of ionization. A pad where energy was deposited is said to be a "hit." This analysis is based on the "subevent" technique wherein one studies the correlation of hits in one part of the detector with the event plane angle as determined by hits in a different part of the detector [13]. As described in the earlier work [1-3,17], corrections are applied to account for signal dilution due to detector occupancy and adjustments are made to create an appropriately symmetric acceptance for the analysis. The subevent regions used in the event plane calculation were $0.1<|\eta|<3.0$ for both 62.4 and $200 \mathrm{GeV}$. The event plane resolution was calculated separately for each centrality bin. The resolution correction factor ranged from 2 to 3 on average, with the larger correction necessary at $62.4 \mathrm{GeV}$. For the determination of $v_{2}$ in the positive (negative) $\eta$ region of the detector, the subevent on the opposite side of midrapidity was used to evaluate $\Psi_{2}$.

Monte Carlo simulations showed a residual suppression of the flow signal dominated by background particles carrying no flow information and the loss of sensitivity due to the hit map symmetrization and the occupancy correction algorithm. As in our earlier work with the hitbased technique, this suppression was corrected using simulated data by comparing the output resolution corrected flow signal to the input flow signal for many samples of simulated data with different shapes and magnitudes of input flow signal.

Numerous sources of systematic error were investigated, including effects due to the hit definition, hit merging, subevent definition, knowledge of the beam orbit relative to the detector, hole filling procedure, vertexing algorithm, and suppression correction determination. The effect of these sources depended both on $\eta$ and centrality. In general, the systematic error arising from each source was determined by varying that specific aspect of the analysis (or several aspects in concert) within reasonable limits and quantifying the change in the final $v_{2}$ result as a function of $\eta$ and centrality. The individual contributions were added in quadrature to derive the $90 \%$ confidence level error shown in the results presented here. The systematic uncertainty was dominated by the suppression correction determination.

Figure 1 shows the elliptic flow signal as a function of pseudorapidity in $\mathrm{Cu}-\mathrm{Cu}$ collisions at $\sqrt{s_{N N}}=62.4$ and $200 \mathrm{GeV}$ for the $40 \%$ most central events. The resemblance to published $\mathrm{Au}-\mathrm{Au}$ results [3] (also shown in Fig. 1) is striking. The $\mathrm{Cu}-\mathrm{Cu} v_{2}$ displays a similar shape in pseudorapidity to that of $\mathrm{Au}-\mathrm{Au}$, with a magnitude at midrapidity only $10 \%-20 \%$ lower than that seen in $\mathrm{Au}-\mathrm{Au}$, increasing to $\sim 40 \%$ at large $|\eta|$. The strength of the $\mathrm{Cu}-$ $\mathrm{Cu} v_{2}$ signal is surprising in light of expectations that the smaller system size would result in a much smaller flow signal [9].

The $\mathrm{Cu}-\mathrm{Cu} v_{2}$ also exhibits extended longitudinal scaling, as shown in Fig. 2, and as already seen in $\mathrm{Au}-\mathrm{Au}$ collisions for elliptic flow [3] and directed flow [17] and for charged particle multiplicity [18-20]. The agreement between the two energies in $|\eta|-y_{\text {beam }}$ implies that, as with $\mathrm{Au}-\mathrm{Au}$, the elliptic flow is largely independent of energy when viewed (effectively) in the rest frame of one of the colliding nuclei.

The centrality dependence of the elliptic flow measured in $\mathrm{Cu}-\mathrm{Cu}$ is presented in Fig. 3, where $v_{2}$ is plotted as a function of the number of participating nucleons, $N_{\text {part }}$. Both hit-based and track-based analyses were used for the $200 \mathrm{GeV}$ data, and the results of the two methods agree quite well within errors.

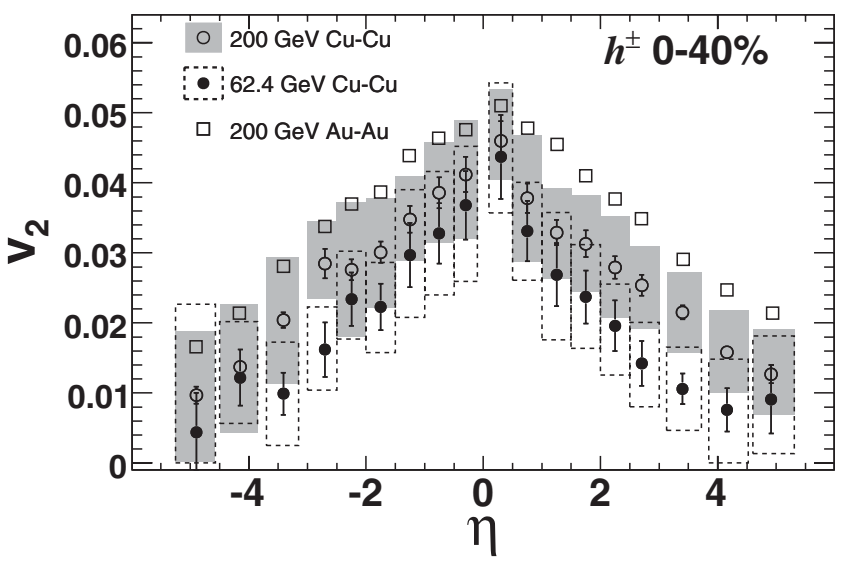

FIG. 1. $v_{2}$ vs $\eta$ for $\mathrm{Cu}-\mathrm{Cu}$ collisions at $\sqrt{s_{N N}}=62.4$ and $200 \mathrm{GeV}$ using the hit-based analysis. The boxes show the $90 \%$ C.L. systematic errors and the bars represent the 1- $\sigma$ statistical errors. Previously published $200 \mathrm{GeV} \mathrm{Au}-\mathrm{Au}$ data (without error bars) are shown for comparison. 


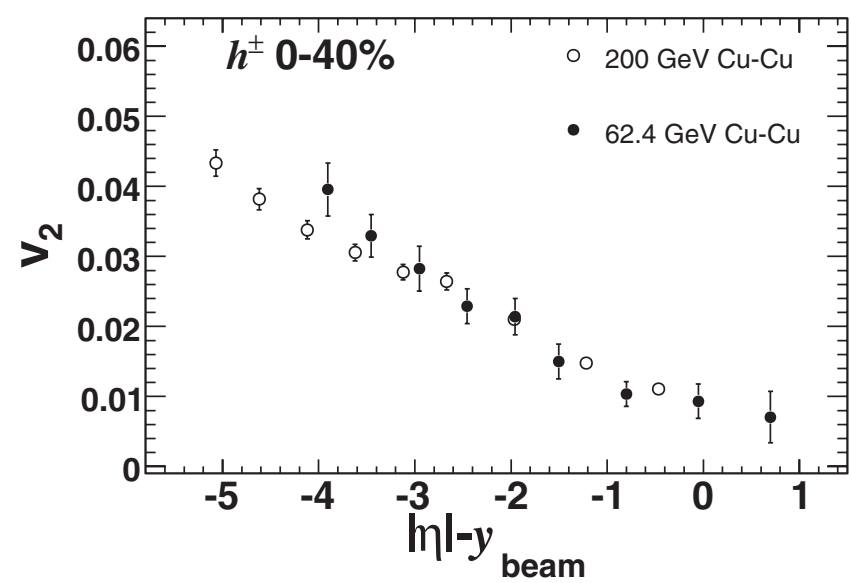

FIG. 2. $v_{2}$ vs $|\eta|-y_{\text {beam }}$ for $\mathrm{Cu}-\mathrm{Cu}$ collisions at $\sqrt{s_{N N}}=62.4$ and $200 \mathrm{GeV}$ from the hit-based analysis. Only $1-\sigma$ statistical errors are shown.

A substantial flow signal is measured in $\mathrm{Cu}-\mathrm{Cu}$ at both energies for even the most central events. This is quite surprising, as the initial spatial anisotropy gives rise to a momentum space anisotropy which, in turn, produces the flow [21]. It is expected therefore that $v_{2}$ should approach zero as the collisions become more central, as it does for $\mathrm{Au}-\mathrm{Au}[2]$. The persistent and nontrivial elliptic flow signal seen in the most central events implies that something beyond the expected nuclear collision geometry may be responsible for driving the flow signal. One natural possibility, discussed for instance in Refs. [22,23], is that fluctuations may become important for some centralities and/ or systems.

To explore this question further, it is useful to compare directly the elliptic flow signal across different colliding species, i.e., make a direct comparison between the flow

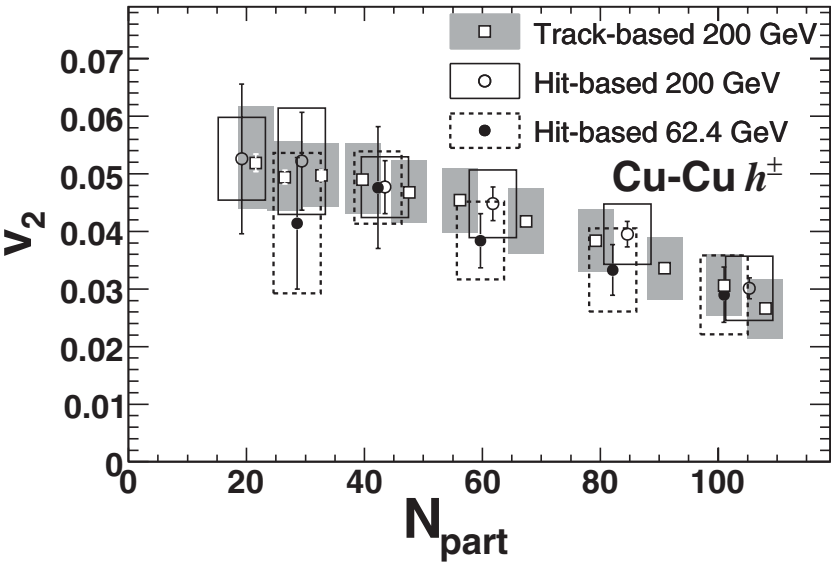

FIG. 3. $v_{2}$ vs $N_{\text {part }}$ for $\mathrm{Cu}-\mathrm{Cu}$ collisions at $\sqrt{s_{N N}}=62.4$ and $200 \mathrm{GeV}$. The boxes show the $90 \%$ C.L. systematic errors and the lines represent the 1- $\sigma$ statistical errors. The results from two analysis methods are shown, as discussed in the text. $v_{2}$ is shown for $|\eta|<1$ and $0<\eta<1$ for the hit-based and track-based methods, respectively. seen in $\mathrm{Cu}-\mathrm{Cu}$ and $\mathrm{Au}-\mathrm{Au}$ collisions. To do this, it is important to scale out the difference in the initial geometric asymmetry of the collision, i.e., the eccentricity of the collision. This is crucial since in each selected centrality range the average eccentricity depends on the size of the colliding species.

Typically, the eccentricity is defined by relating the impact parameter of the collision in a Glauber model simulation to the eccentricity calculated assuming the minor axis of the overlap ellipse to be along the impact parameter vector. Thus, if the $x$ axis is defined to be along the impact parameter vector and the $y$ axis perpendicular to that in the transverse plane, the eccentricity is determined by $[24,25]$

$$
\varepsilon=\frac{\sigma_{y}^{2}-\sigma_{x}^{2}}{\sigma_{y}^{2}+\sigma_{x}^{2}}
$$

where $\sigma_{x}$ and $\sigma_{y}$ are the rms widths of the participant nucleon distributions projected on the $x$ and $y$ axes, respectively. Let us call the eccentricity determined in this fashion $\varepsilon_{\text {std }}$.

The relation of the eccentricity to the centrality depends on the details of the eccentricity definition used in the Glauber model simulation. The definition most commonly used is presented above. Implicit in this choice is a physics bias about the relevant asymmetry that drives the flow signal. It is important to consider other possibilities. In particular, a natural choice to consider is the geometry of the participant nucleons themselves.

In a large system, the nuclear geometry and the participant geometry largely coincide. For small systems or small transverse overlap regions, however, fluctuations in the nucleon positions in Glauber model calculations, as described below, frequently create a situation where the minor axis of the ellipse in the transverse plane formed by the participating nucleons is not along the impact parameter vector. One way to address this issue is to make a principal axis transformation, rotating the $x$ and $y$ axes used in the eccentricity definition in the transverse plane in such a way that $\sigma_{x}$ is minimized. Let us call the eccentricity determined in this fashion the participant eccentricity, $\varepsilon_{\text {part }}$, and the plane specified by the beam axis and the $x$ axis in the participant frame $\Psi_{\text {part }}$. In terms of the original $x$ and $y$ axes (in fact, any pair of perpendicular transverse axes),

$$
\varepsilon_{\mathrm{part}}=\frac{\sqrt{\left(\sigma_{y}^{2}-\sigma_{x}^{2}\right)^{2}+4\left(\sigma_{x y}\right)^{2}}}{\sigma_{y}^{2}+\sigma_{x}^{2}} .
$$

In this formula, $\sigma_{x y}=\langle x y\rangle-\langle x\rangle\langle y\rangle$. The average values of $\varepsilon_{\text {std }}$ and $\varepsilon_{\text {part }}$ are quite similar for all but the most peripheral interactions for large species, as is shown in Fig. 4 for Au$\mathrm{Au}$. For smaller systems such as $\mathrm{Cu}-\mathrm{Cu}$, however, fluctuations in the nucleon positions in Glauber model calculations (described below) become quite important for all centralities and the average eccentricity can vary signifi- 
cantly depending on how it is calculated. This is also illustrated in Fig. 4. It should be noted that the behavior of the eccentricities shown in Fig. 4 for $N_{\text {part }}$ below $\sim 19$ $(\sim 55)$ for $\mathrm{Cu}-\mathrm{Cu}(\mathrm{Au}-\mathrm{Au})$ are shown only to display the mathematical behavior of the two definitions of the eccentricity, since data are unavailable in these ranges. However, it is interesting that $\varepsilon_{\text {std }}$ becomes negative at low $N_{\text {part }}$ for both $\mathrm{Cu}-\mathrm{Cu}$ and $\mathrm{Au}-\mathrm{Au}$ due to fluctuations, while $\varepsilon_{\text {part }}$ by definition must be positive.

The effects of finite number and eccentricity fluctuations on elliptic flow have been studied for large collision systems with Monte Carlo simulations [23,26,27] and were found to be small. However, in $\mathrm{Cu}-\mathrm{Cu}$ collisions these fluctuations are larger and could have a significant impact on the elliptic flow. The participant eccentricity allows these fluctuating configurations to be considered seriously on an event-by-event basis.

The Glauber model used for the calculation of these eccentricities is a Monte Carlo toy model that builds nuclei by randomly placing nucleons according to a WoodsSaxon distribution. Excluded volume effects were incorporated into the model, requiring a minimum center-to-center nucleon separation of $0.4 \mathrm{fm}$, to agree with HIJING [15]. A number of sources of systematic error were studied, including nuclear radius, nuclear skin depth, nucleonnucleon inelastic cross section $\sigma_{N N}$, and minimum nucleon separation. The systematic error contributed by each source was determined by varying that specific parameter in the analysis within reasonable limits and quantifying the change in the final eccentricity result as a function of centrality. The individual contributions were added in quadrature to determine the $90 \%$ confidence level errors shown in Fig. 4.

The crucial importance of the definition of eccentricity in comparing $\mathrm{Cu}-\mathrm{Cu}$ and $\mathrm{Au}-\mathrm{Au}$ results for a given beam

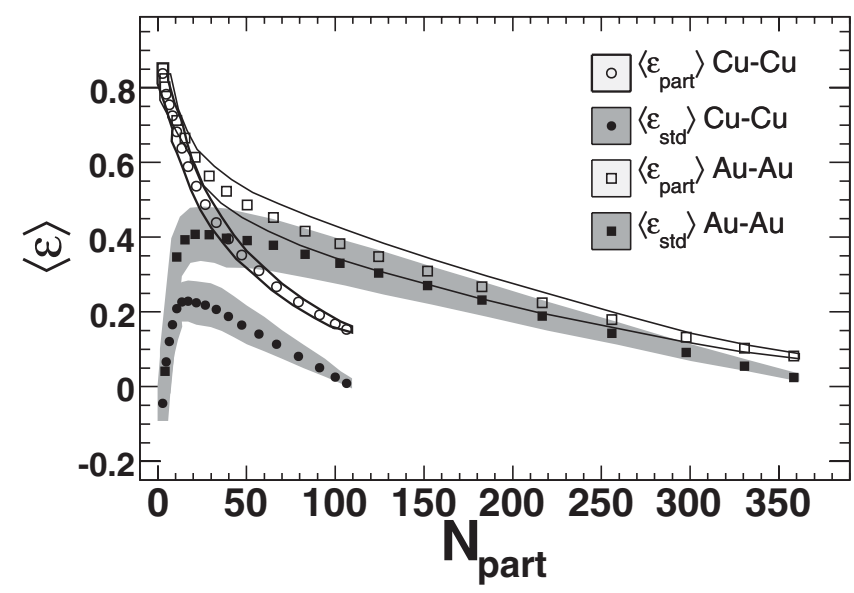

FIG. 4. The average eccentricity defined in two ways, $\left(\left\langle\varepsilon_{\text {std }}\right\rangle\right.$ and $\left.\left\langle\varepsilon_{\text {part }}\right\rangle\right)$, as described in the text, versus $N_{\text {part }}$ for simulated $\mathrm{Cu}-\mathrm{Cu}$ and $\mathrm{Au}-\mathrm{Au}$ collisions at $\sqrt{s_{N N}}=200 \mathrm{GeV}$. The bands show the $90 \%$ C.L. systematic errors. energy can be seen in Fig. 5, where comparisons are made between $\mathrm{Cu}-\mathrm{Cu}$ and $\mathrm{Au}-\mathrm{Au}$ data at 62.4 and $200 \mathrm{GeV}$ using the eccentricity-scaled elliptic flow. These comparisons are made as a function of the number of participating nucleons since $N_{\text {part }}$ is a well-understood quantity that is directly related to the centrality and the calculated eccentricities in the Glauber model. In Fig. 5(b), $v_{2} / \varepsilon_{\text {std }}$ increases rapidly in $\mathrm{Cu}-\mathrm{Cu}$ as the events become more central, and is generally larger than that of $\mathrm{Au}-\mathrm{Au}$. One might then conclude from this that either the smaller $\mathrm{Cu}-\mathrm{Cu}$ system produces $v_{2}$ much more efficiently than the larger Au-Au system or that $\varepsilon_{\text {std }}$ may not be the appropriate quantity for describing the initial geometry of the collision. Consider then Fig. 5(c), in which $v_{2} / \varepsilon_{\text {part }}$ is shown to be very similar for both $\mathrm{Cu}-\mathrm{Cu}$ and $\mathrm{Au}-\mathrm{Au}$ at the same beam energy, even appearing to lie on the same curve. Given the qualitative and quantitative similarities between the results in the two systems already shown, it is not unreasonable to expect both systems to have a similar eccentricity-scaled elliptic flow, as in Fig. 5(c). Therefore, it seems likely that $\varepsilon_{\text {part }}$ as discussed here and in Ref. [28] — or a rather similar quantity, such as $\sqrt{\left\langle\varepsilon_{\text {part }}^{2}\right\rangle}[29]$ - is the relevant eccentricity for the azimuthal anisotropy.

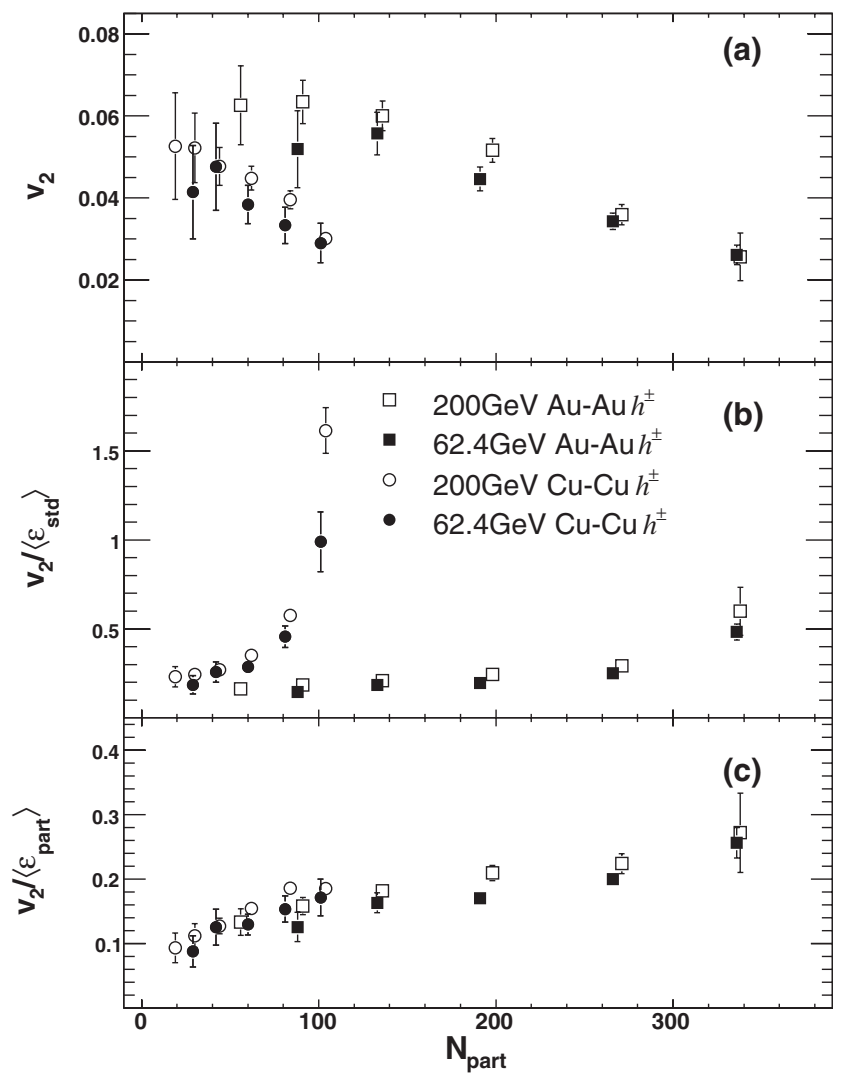

FIG. 5. (a) $v_{2}$ (unscaled) vs $N_{\text {part }}$, (b) $v_{2} /\left\langle\varepsilon_{\text {std }}\right\rangle$ vs $N_{\text {part }}$, and (c) $v_{2} /\left\langle\varepsilon_{\text {part }}\right\rangle$ vs $N_{\text {part }}$, for $\mathrm{Cu}-\mathrm{Cu}$ and $\mathrm{Au}-\mathrm{Au}$ collisions at $\sqrt{s_{N N}}=62.4$ and $200 \mathrm{GeV}$. 1- $\sigma$ statistical error bars are shown. $v_{2}$ is shown in $|\eta|<1$ for the hit-based method. 
In summary, the results presented here show a measurable and significant elliptic flow signal in $\mathrm{Cu}-\mathrm{Cu}$ collisions at 62.4 and $200 \mathrm{GeV}$. These data show that qualitative features attributed to collective effects in $\mathrm{Au}-\mathrm{Au}$ persist down to the relatively small numbers of participants seen in the $\mathrm{Cu}-\mathrm{Cu}$ collision and are of comparable magnitude. The essential role of the choice of collision geometry in comparing flow across nuclear species is clearly demonstrated. If it is assumed that the flow is independent of species for a given collision geometry, the data shown here strongly suggest that it is the participant eccentricity, not the standard (nuclear) eccentricity, that is responsible for elliptic flow. These results also imply that $\Psi_{2}$ is an estimate of $\Psi_{\text {part }}$, not $\Psi_{R}$, which seems to define the orientation for the $v_{2}$ signal, particularly for systems with smaller numbers of participants. This, in turn, may provide information on the nature of the matter driving the flow.

This work was partially supported by U.S. DOE Grants No. DE-AC02-98CH10886, No. DE-FG02-93ER40802, No. DE-FC02-94ER40818, No. DE-FG02-94ER40865, No. DE-FG02-99ER41099, and No. DE-AC0206CH11357, U.S. NSF Grants No. 9603486 , No. 0072204, and No. 0245011, Polish KBN Grant No. 1-P03B-062-27(2004-2007), NSC of Taiwan Contract No. NSC 89-2112-M-008-024, and Hungarian OTKA Grant (No. F 049823).

[1] B. B. Back et al., Phys. Rev. Lett. 89, 222301 (2002).

[2] B. B. Back et al., Phys. Rev. C 72, 051901 (2005).

[3] B. B. Back et al., Phys. Rev. Lett. 94, 122303 (2005).
[4] C. Adler et al., Phys. Rev. C 66, 034904 (2002).

[5] J. Adams et al., Phys. Rev. Lett. 93, 252301 (2004).

[6] J. Adams et al., Phys. Rev. C 72, 014904 (2005).

[7] S. S. Adler et al., Phys. Rev. Lett. 91, 182301 (2003).

[8] S. S. Adler et al., Phys. Rev. Lett. 94, 232302 (2005).

[9] L-W. Chen and C. M. Ko, Phys. Lett. B 634, 205 (2006).

[10] A. Adams et al., nucl-ex/0608033.

[11] S. Voloshin for the STAR Collaboration, nucl-ex/0610038.

[12] T. Hirano et al., Phys. Lett. B 636, 299 (2006).

[13] A. M. Poskanzer and S. A. Voloshin, Phys. Rev. C 58, 1671 (1998).

[14] B. B. Back et al., Nucl. Instrum. Methods Phys. Res., Sect. A 499, 603 (2003).

[15] M. Gyulassy and X. N. Wang, Phys. Rev. D 44, 3501 (1991). We used standard HIJING v1.35.

[16] GEANT 3.211, CERN Program Library.

[17] B. B. Back et al., Phys. Rev. Lett. 97, 012301 (2006).

[18] I. G. Bearden et al., Phys. Rev. Lett. 88, 202301 (2002).

[19] B. B. Back et al., Phys. Rev. Lett. 91, 052303 (2003).

[20] B. B. Back et al., Phys. Rev. C 74, 021901 (2006).

[21] W. Reisdorf and H. G. Ritter, Annu. Rev. Nucl. Part. Sci. 47, 663 (1997).

[22] J. Y. Ollitrault, Phys. Rev. D 48, 1132 (1993).

[23] M. Miller and R. Snellings, :nucl-ex/0312008.

[24] H. Heiselberg and A-M. Levy, Phys. Rev. C 59, 2716 (1999).

[25] H. Sorge, Phys. Rev. Lett. 82, 2048 (1999).

[26] J-Y. Ollitrault, Phys. Rev. D 46, 229 (1992).

[27] X. Zhu, M. Bleicher, and H. Stöcker, Phys. Rev. C 72, 064911 (2005).

[28] S. Manly for the PHOBOS Collaboration, Nucl. Phys. A774, 523 (2006).

[29] R. S. Bhalerao and J-Y. Ollitrault, Phys. Lett. B 641, 260 (2006). 